\title{
Psychophysical parameters of the perception of orally-retained liquid bulk
}

\author{
R. A. M. GREGSON AND HELEN M. MATTERSON \\ UNIVERSITY OF CANTERBURY, NEW ZEALAND
}

Bulk of liquid held in the mouth may be judged on its subjective volume. Over the range $1 \mathrm{ml}-31 \mathrm{ml}$, scaling by the constant method and by ratio estimation yielded a Weber fraction which was not constant and decreased with stimulus magnitudes and a psychophysical power function with an exponent of 0.96 . A "negative thre shold" effect is reported.

The human capacity to hold liquid samples in the mouth without swallowing is accompanied by a limited ability to judge the relative bulk of such samples. Clearly, such judgments are mediated physiologically in a complex fashion. Cues to facilitate judgment are available from the weight, volume, viscosity, temperature, and taste of liquids, and also from resilience in the case of colloidal-phase stimuli (Szczesniak \& Farkas, 1962). Under normal circumstances liquid samples are not weightless, and may not be tasteless. It is relatively easy to prepare tasteless samples, but without entering zero-gravity conditions weightless liquids are impractical. Therefore the term "liquid bulk" is used here to refer to the complex of sensations which result from holding a mass of tasteless liquid, of specified density, viscosity and temperature, in the mouth. Some cues of volume and weight are available to Ss, and feelings resulting from tongue movements in liquid and consequent liquid flow through the interstices of the teeth may provide further cues. No attempt here has been made to distinguish information from these different potential sources; the psychophysics of the normal unimpeded perception of liquid bulk as a gestalt quality is being investigated. The effective discriminability of water volume differences, and the psychophysical power function (Stevens, 1961) relating perceived to actual volume (or weight) for water have been estimated.

\section{Method}

Subjects. Thirty-six persons from the university campus, 18 males and 18 females, mainly first-year psychology undergraduates.

Stimuli. Deionised water samples (equivalent to triple distilled in purity) with resistance not less than $2 \mathrm{M} \Omega / \mathrm{cm}$ were measured out from burettes into $50 \mathrm{ml}$ beakers. The beakers were coated with opaque black and weighted inside with lead shot embedded in wax, to a varying unladen total varying between 95 and $105 \mathrm{gm}$. Thus the stimuli did not furnish consistent or facile visual or kinesthetic cues for Ss to guess volumes in advance of ingesting them.
Procedure. The constant method (Guilford, 1954) and a paired comparison ratio scaling method (Comrey, 1950; Torgerson, 1958) were both used as two separate tasks, in a design balanced for order effects within and between tasks. Each S attended over three sessions. At each session the constant method with one standard, and a one-third part of the paired comparison matrix were completed.

For the constant method, the volumetric series were (a) standard $5 \mathrm{ml}$, stimuli $3,4,5,6$ and $7 \mathrm{ml}$, (b) standard $15 \mathrm{ml}$, stimuli $12,13.5,15,16.5$ and $18 \mathrm{ml}$, (c) standard $25 \mathrm{ml}$, stimuli $21,23,25,27$ and $29 \mathrm{ml}$. Each $\mathrm{S}$ did all comparisons with a standard in both temporal orders once, making 10 paired comparisons for each standard. Overall results are thus based on the means of 72 observations per comparison.

For the ratio scaling method, the stimulus volumes paired were (in $\mathrm{ml}$ ) (d) $16-6,31-11,21-1,26-16$, $6-31,11-1,16-21$, (e) $26-31,1-6,21-26,11-16,31-1$, $26-6,21-11$, (f) 16-31, 1-26, 6-11, 31-21, 1-16, 11-26, 6-21. Ss were instructed to partition an imaginary line 100 units long in the proportion of the volumes compared, giving the proportion of 100 representing the second stimulus in each pair. All paired comparisons in (a) through (f) were made to a fixed time schedule, signalled to $\mathrm{S}$ by a buzzer. Individual stimuli were held in the mouth for 3 sec., and 5 sec. elapsed between the stimuli in each pair. Ss were used singly, seated in the cubicle of the Gustatory Psychophysics laboratory at the University of Canterbury. There was no illumination provided in the cubicle, and tasting was in almost complete darkness. $E$ was consequently not visible to $S$ during the experiment.

\section{Results}

Trial analysis showed that differences between sexes were very slight; results have thus been pooled over all $36 \mathrm{Ss}$.

Fitting linear regressions to the normalized mean probability of "greater volume" responses against physical volume in $\mathrm{ml}$, Table 1 was obtained. Each column refers to one condition of (a) through (c).

Scaling the ratio judgments by Comrey's (1950) method gave

$$
\psi=.088(\phi+0.45)
$$

as fitted equation when $\phi$ is physical volume in and $\psi$ is in arbitrary sensation magnitudes. The linear regression takes up $99 \%$ of the variance of the scaled values. 
Table 1. Psychophysical parameters of liquid bulk obtained from the constant method.

\begin{tabular}{|c|c|c|c|}
\hline & (a) & (b) & (c) \\
\hline Standard in $\mathrm{ml}$ & 5 & 15 & 25 \\
\hline Constant error & .2 & .26 & .3 \\
\hline Difference limen & 1.0 & 1.75 & 2.2 \\
\hline P. S. E. & 4.0 & $\begin{array}{r}15.26 \\
12\end{array}$ & $\begin{array}{l}25.3 \\
.09\end{array}$ \\
\hline $\begin{array}{l}\text { Weber fraction } \\
\% \text { Variance taken up } \\
\text { by regression }\end{array}$ & $98^{.21}$ & $99^{.12}$ & $96^{.09}$ \\
\hline
\end{tabular}

A psychophysical power function, estimated from all the 21 ratios of (d) through (f) considered together, with iterative graphical estimation of the "threshold" correction to give linearity, yielded

$$
\psi=1.17(\phi+0.25)^{0.96}
$$

as best linear fit, taking up $92 \%$ of the variance of the 21 geometric means of subjective ratios, using a $\log \phi-\log \psi$ plot.

\section{Discussion}

Liquid bulk retained in the mouth is scaleable like many other psychophysical dimensions: none of the parameters have unusually large or small magnitudes considering the nature of the judgment task involved.

The decreasing Weber fraction with increasing standard, contraindicating Weber's law, is consistent with results in some other modalities, particularly the chemical senses (Holway \& Pratt, 1936). The Weber fraction values are smaller than those usually reported for taste, thus emphasising the necessity of keeping volumes of liquid taste stimuli constant in gustatory comparison experiments to avoid providing a basis for a response bias or taste pseudo-discrimination.

The "threshold" constant, for linear regression fitting, is seen to be positive in both methods used.
This implies that a sensory magnitude exists when there is no input to the judgment process, or that a constant is added to all stimulus magnitudes by the response system at the moment of stimulus presentation; a sort of negative threshold effect. Residual salfva, or a change in salivation rate consequent upon ingestion of liquid could underlie this effect. The actual correction is equivalent in the power function to about a quarter of a gram, which is certainly neither the exact weight of saliva usually in the mouth nor the effective weight to be moved when the tongue is agitater to assess the occupancy of the oral cavity.

The psychophysical power law exponent is very near to unity, as is the case for a number of other continua examined by Stevens and his co-workers. This psychophysical continuum is unusual in one respect, in that both its ends are physically defined by simple mechanical considerations. Either no liquid is introduced into the mouth, or there is so much that involuntary swallowing eventually ensues. The upper limit of oral liquid capacity voluntarily retained is not far above the top value of $31 \mathrm{ml}$ used here: extrapolation upwards of any of the trends reported does not therefore appear justified.

\section{References}

Comrey, A. L. A proposed method for absolute ratio scaling. Psychometrika, 1950, 15, 317-325.

Guilford, J. P. Psychometric methods. (2nd ed.) New York: McGraw Hill, 1954.

Holway, A. H., \& Pratt, C. C. The Weber-ratio for intensive discrimination. Psychol. Rev., 1936, 43, 322-340.

Stevens, S. S. The psychophysics of sensory function. In W. A. Rosenblith (Ed.), Sensory communication. New York: Wiley, 1961. Pp. 1-33.

Szczesniak, Alina S., \& Farkas, Elizabeth. Objective characterization of the mouthful of gum solutions. J. Food Sci., 1962, 27 381-385.

Torgerson, W. S. Theory and methods of scaling. New York: Wiley, 1958.

(Accepted for publication December 9, 1966.) 